\title{
Lipid and inflammatory biomarkers and kidney function decline in type 2 diabetes
}

\author{
J. Lin • F. B. Hu • C. Mantzoros • G. C. Curhan
}

Received: 18 June 2009 / Accepted: 8 October 2009 / Published online: 18 November 2009

(C) Springer-Verlag 2009

\begin{abstract}
Aims/hypothesis Potentially modifiable biomarkers may influence the decline in estimated GFR (eGFR), but few data are currently available in type 2 diabetic adults.

Methods We studied 516 women with type 2 diabetes in the Nurses' Health Study with data on lipid and inflammatory biomarkers from plasma collected in 1989 and plasma creatinine in samples collected in 1989 and 2000. An estimated GFR decline of $\geq 25 \%$ over 11 years was the outcome of interest.

Results Comparing the highest with the lowest quartile, soluble tumour necrosis factor receptor 2 (sTNFR-2) was
\end{abstract}

Electronic supplementary material The online version of this article (doi:10.1007/s00125-009-1597-z) contains supplementary material, which is available to authorised users.

J. Lin $(\bowtie) \cdot$ G. C. Curhan

Renal Division, MRB-4, Brigham and Women's Hospital,

75 Francis Street,

Boston, MA 02115, USA

e-mail: jlin11@partners.org

J. Lin · F. B. Hu • G. C. Curhan

Channing Laboratory, Department of Medicine,

Brigham and Women's Hospital, Harvard Medical School,

Boston, MA, USA

F. B. $\mathrm{Hu} \cdot$ G. C. Curhan

Department of Epidemiology, Harvard School of Public Health,

Boston, MA, USA

F. B. Hu

Department of Nutrition, Harvard School of Public Health,

Boston, MA, USA

\section{Mantzoros}

Division of Endocrinology, Department of Medicine,

Beth Israel Deaconess Medical Center,

Boston, MA, USA independently associated with an eGFR decline of $\geq 25 \%$ (multivariate OR 5.81; 95\% CI 2.90-11.65); this association was stronger in obese women (OR 16.76; 95\% CI 4.69-59.90 for $\mathrm{BMI} \geq 30 \mathrm{~kg} / \mathrm{m}^{2}$; OR $2.78,95 \%$ CI 1.12 6.89 for $\mathrm{BMI}<30 \mathrm{~kg} / \mathrm{m}^{2} ; p$ for interaction $=0.02$ ). No lipids (total cholesterol, LDL-cholesterol, HDL-cholesterol, nonHDL-cholesterol, triacylglycerols, lipoprotein(a), or apolipoprotein B) or other markers of inflammation (C-reactive protein, fibrinogen, E-selectin, intracellular cell adhesion molecule 1, leptin or adiponectin) were significantly associated with eGFR decline after multivariable adjustment. Conclusions/interpretation Elevated sTNFR-2 levels may be an important and potentially modifiable risk factor for eGFR decline in type 2 diabetes, especially in those with a BMI of $\geq 30 \mathrm{~kg} / \mathrm{m}^{2}$.

Keywords Estimated GFR decline - Inflammation · Kidney function decline $\cdot$ Lipids · sTNFR-2 Type 2 diabetes

$\begin{array}{ll}\text { Abbreviations } \\ \text { ACE-I } & \text { Angiotensin-converting enzyme inhibitor } \\ \text { CARE } & \text { Cholesterol and Recurrent Events } \\ \text { CRP } & \text { C-reactive protein } \\ \text { DSQ } & \text { Diabetes supplementary questionnaire } \\ \text { eGFR } & \text { Estimated GFR } \\ \text { NHS } & \text { Nurses' Health Study } \\ \text { sTNFR-2 } & \text { Soluble tumour necrosis factor receptor-2 }\end{array}$

\section{Introduction}

Identifying biomarkers predictive of estimated GFR (eGFR) decline could point to pathways that may help identify new therapeutic agents. With the recognition that type 2 diabetes itself may be a chronic inflammatory state, the role of 
potentially modifiable lipid and inflammatory biomarkers has been of particular interest.

Notably, higher baseline C-reactive protein (CRP) was associated with eGFR decline in people with hyperlipidaemia and a history of myocardial infarction [1] and in older community-dwelling adults [2]. No relation between CRP and eGFR decline was identified, however, in an analysis of the Modification of Diet in Renal Disease study [3], but mean duration of follow-up was only 2.2 years. Soluble tumour necrosis factor receptor 2 (sTNFR-2) has also been associated with eGFR decline [1]. These investigations included less than $15 \%$ diabetic patients, however.

We previously reported that dyslipidaemia, as well as higher fibrinogen, vascular cell adhesion molecule 1 and sTNFR-2, were independently associated with lower eGFR in a cross-sectional study of men with type 2 diabetes [4]. In this study we expanded our investigations by examining the relation between lipid and inflammatory biomarkers with respect to the 11-year eGFR decline in women with type 2 diabetes.

\section{Methods}

This study was approved by the Partners' Healthcare Brigham and Women's Hospital Human Research Committee Institutional Review Board.

Participants The Nurses' Health Study (NHS) was initiated in 1976 with the enrolment of 121,700 US nurses aged 3055 years. Questionnaires are mailed biennially; return of questionnaires constitutes informed consent under the study's institutional review board protocol. Between 1989 and 1990, 32,826 participants provided blood samples that were shipped on ice by overnight delivery and stored at $-130^{\circ} \mathrm{C}$ as previously described [5]. In the year 2000, many of these participants submitted a second blood sample under the same shipment and storage conditions.

We mailed a diabetes supplementary questionnaire (DSQ) to all women reporting diabetes on biennial questionnaires. We used the National Diabetes Data Group criteria [6] to define diabetes self-reported up to the 1996 biennial questionnaire. Self-reported diagnosis using the DSQ was $98 \%$ accurate in a validation study with medical records review [7].

We included women who reported being diagnosed with type 2 diabetes up to the year 2000 (because glucose intolerance can precede the diagnosis of type 2 diabetes by $\geq 10$ years [8]) and who had plasma samples from both 1989 and 2000. We excluded women with a serum creatinine concentration $\leq 44 \mathrm{mmol} / 1$ (felt to be implausible) $(n=12)$ and those for whom data on plasma creatinine were missing $(n=19)$. After applying these criteria, 516 women had eGFR change data available for analysis (Fig. 1).

Biomarker analyses Biomarker assays were performed at Children's Hospital, Boston, MA, USA, or in the laboratory of Dr C. Mantzoros at Beth Israel Deaconess Hospital, Boston, MA, USA, using standard methods and assays (see Electronic supplementary material). On blinded quality control samples that were run simultaneously with participant samples, biomarker measurements had CVs of less than $15 \%$ with the exception of E-selectin (20\%), adiponectin $(27.4 \%)$ and sTNFR-2 (16.7\%).

Measurements of kidney function decline Plasma creatinine was analysed using a modified kinetic Jaffe reaction $(\mathrm{CV}$ $10 \%$ ). In 2007 , repeat creatinine assays of 20 NHS plasma samples (with a range of 53 to $124 \mathrm{mmol} / \mathrm{l}$ ) initially measured in the year 2000 revealed a mean recalibration coefficient (new value/original value) of 0.97 and confirmed that creatinine was stable under our storage conditions.

Glomerular filtration was estimated by the abbreviated MDRD (Modification of Diet in Renal Disease) equation [9]. An eGFR decline of $\geq 25 \%$ between 1989 and 2000 was the primary outcome. An eGFR decline of $\geq 25 \%$ over 11 years was determined a priori and had been used in previous analyses of renal function decline in NHS participants [10].

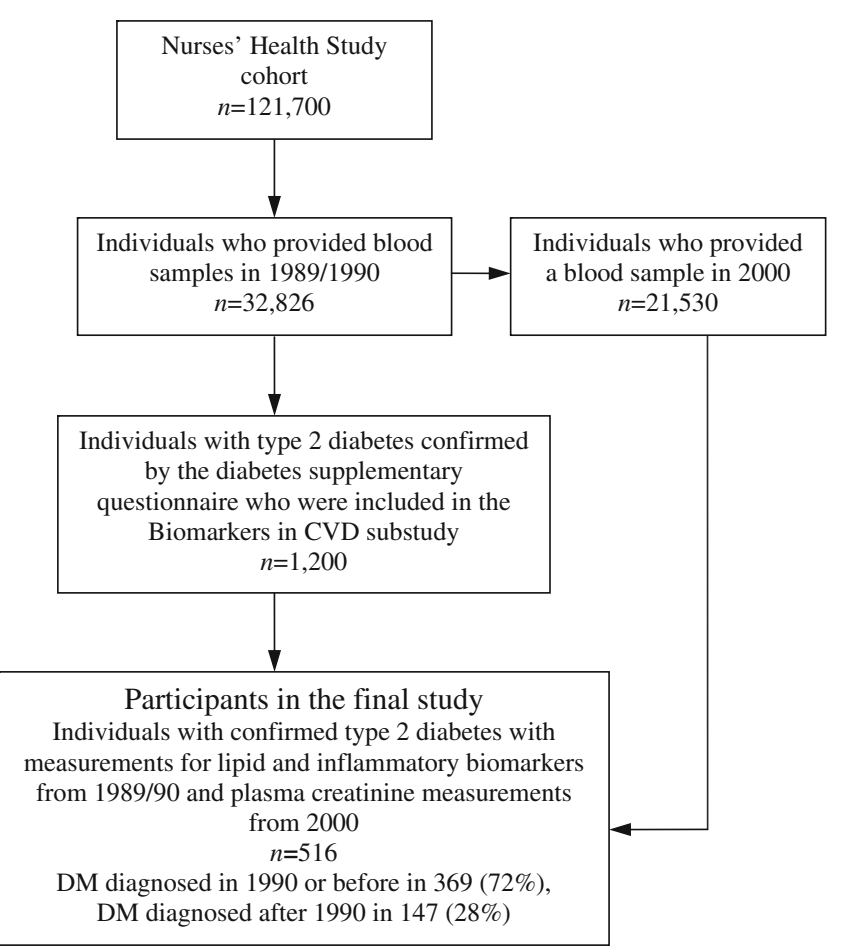

Fig. 1 Biomarkers and type 2 diabetes participant selection in the Nurses' Health Study. DM, diabetes mellitus 
Statistical analysis Spearman correlations were calculated for eGFR and individual biomarkers at baseline. Multivariable logistic regression modelling was applied to analyse associations between biomarkers and an eGFR decline of $\geq 25 \%$. Animal protein intake, alcohol intake and baseline cardiovascular disease were also considered for the adjusted models but were removed as they did not change results. Analyses were performed with SAS software, version 9.1 (SAS Institute, Cary, NC, USA).

\section{Results}

Characteristics of the study cohort are summarised in Table 1. In 1989, significant modest cross-sectional inverse correlations were noted between eGFR and leptin $(r=0.16$, $p<0.001)$, adiponectin $(r=-0.13, p=0.003)$ and sTNFR-2 $(r=-0.28, p<0.001)$. CRP was not associated with eGFR $(r=0.03, p=0.57)$. The women who experienced an eGFR decline of $\geq 25 \%$ between 1989 and 2000 had higher rates of hypertension, cardiovascular disease, angiotensinconverting enzyme inhibitor (ACE-I)/angiotensin-type 2 receptor blocker medication use, and lower physical activity. In 1989, only 35 women (6.8\%) had eGFR $<60 \mathrm{ml} \mathrm{min}{ }^{-1} 1.73 \mathrm{~m}^{-2}$. In 2000, 139 women $(27 \%)$ had eGFR $<60 \mathrm{ml} \mathrm{min}^{-1} 1.73 \mathrm{~m}^{-2}$.

Those in the highest quartile of sTNFR-2 in 1989 had significantly higher odds for an eGFR decline of $\geq 25 \%$ between 1989 and 2000 (multivariate OR 5.81; 95\% CI
2.90-11.65) (Table 2 and Fig. 2). The association between sTNFR-2 and eGFR decline was stronger in women with a BMI of $\geq 30$ (OR 16.76; 95\% CI 4.69-59.9) than in women with a BMI of $<30$ (OR 2.78; 95\% CI 1.12-6.89) ( $p$ for interaction $=0.02$ ). No other significant associations between biomarkers and eGFR decline were present after multivariable adjustment. Restricting the analyses to the 369 women diagnosed with diabetes in 1990 or before did not change the results meaningfully.

\section{Discussion}

After multivariable adjustment, sTNFR-2 was a strong predictor of eGFR decline and varied with obesity status. These data support the theory that sTNFR-2, our surrogate marker for the volatile cytokine TNF- $\alpha$, promotes tubular epithelial apoptosis [11] and progressive renal tubulointerstitial fibrosis [12]. Tubulointerstitial fibrosis is one of the strongest predictors of subsequent kidney disease progression [13], which ultimately manifests as GFR decline. Our findings are consistent with the Cholesterol and Recurrent Events (CARE) trial, in which significantly faster eGFR decline was associated with higher levels of sTNFR-2 [1]. Unlike the CARE study, however, we did not see an association between CRP and eGFR decline. This may be attributable to the difference in populations (type 2 diabetic vs mostly non-diabetic individuals with a history of myocardial infarction).
Table 1 Demographic and clinical characteristics of participants of the Nurses' Health Study with type 2 diabetes in year 2000

Data are percentage or median ${ }^{*} p<0.05$ vs referent group (no eGFR decline)

ARB, angiotensin-2 receptor blocker; MET, metabolicequivalent score

\begin{tabular}{|c|c|c|}
\hline \multirow[t]{2}{*}{ Characteristic } & \multicolumn{2}{|c|}{ eGFR decline $\geq 25 \%$} \\
\hline & No $(n=346)$ & Yes $(n=170)$ \\
\hline Age (years) & 68 & 70 \\
\hline White $(\%)$ & 98 & 97 \\
\hline Hypertension (\%) & 73 & $86^{*}$ \\
\hline Years since diabetes diagnosis & 13.9 & 16.1 \\
\hline $\mathrm{HbA}_{1 \mathrm{c}}$ in $1989(\%)$ & 6.1 & $6.5^{*}$ \\
\hline Current smoker $(\%)$ & 6.6 & 4.1 \\
\hline Ever smoked (\%) & 51 & 54 \\
\hline Alcohol intake (g/day) & 0.52 & 0.21 \\
\hline Physical activity level (METs/week) & 8.6 & $4.2^{*}$ \\
\hline Cardiovascular disease $(\%)$ & 19 & $37 *$ \\
\hline BMI in $1988\left(\mathrm{~kg} / \mathrm{m}^{2}\right)$ & 29.0 & 29.3 \\
\hline BMI in $2000\left(\mathrm{~kg} / \mathrm{m}^{2}\right)$ & 29.0 & 30.1 \\
\hline Change in BMI between 1988 and $2000\left(\mathrm{~kg} / \mathrm{m}^{2}\right)$ & 0.0 & 0.0 \\
\hline ACE inhibitor or ARB medication use (\%) & 27 & $36^{*}$ \\
\hline Plasma creatinine in $1989(\mathrm{mmol} / \mathrm{l})$ & 68 & $63 *$ \\
\hline Plasma creatinine in $2000(\mathrm{mmol} / \mathrm{l})$ & 70 & $92 *$ \\
\hline eGFR in $1989\left(\mathrm{ml} \mathrm{min} \min ^{-1} 1.73 \mathrm{~m}^{-2}\right)$ & 81 & $91 *$ \\
\hline eGFR in $2000\left(\mathrm{ml} \mathrm{min} \min ^{-1} 1.73 \mathrm{~m}^{-2}\right)$ & 77 & $56^{*}$ \\
\hline
\end{tabular}


Table 2 Logistic regression models for odds ratios for an eGFR decline of $\geq 25 \%$ between 1989 and 2000 for quartile 4 vs quartile 1 of lipid and inflammatory biomarkers

\begin{tabular}{|c|c|c|c|}
\hline Biomarker & $\begin{array}{l}\text { Age-adjusted univariate model } \\
(n=516) \\
\text { OR for quartile } 4 \text { vs quartile } \\
1(95 \% \mathrm{CI})\end{array}$ & $\begin{array}{l}\text { Full model } \\
\text { OR for quartile } 4 \text { vs quartile } \\
1(95 \% \mathrm{CI})\end{array}$ & $\begin{array}{l}p \text { value for trend across quartiles } \\
\text { for full model }\end{array}$ \\
\hline Total cholesterol (mmol/1) & $1.48(0.87,2.54)$ & $1.26(0.70,2.28)$ & 0.41 \\
\hline LDL-cholesterol (mmol/l) & $1.74(1.02,2.96)$ & $1.36(0.76,2.45)$ & 0.24 \\
\hline HDL-cholesterol (mmol/l) & $0.89(0.52,1.51)$ & $0.94(0.51,1.74)$ & 0.66 \\
\hline Non-HDL-cholesterol (mmol/l) & $1.78(1.03,3.09)$ & $1.39(0.74,2.58)$ & 0.32 \\
\hline Triacylglycerols (mmol/l) & $1.32(0.78,2.23)$ & $1.12(0.62,2.03)$ & 0.60 \\
\hline Lipoprotein(a) ( $\mu \mathrm{mol} / \mathrm{l})$ & $1.19(0.70,2.04)$ & $1.11(0.61,1.99)$ & 0.49 \\
\hline Apolipoprotein B (g/l) & $2.05(1.20,3.53)$ & $1.61(0.87,2.99)$ & 0.24 \\
\hline CRP (mg/l) & $1.43(0.86,2.38)$ & $1.34(0.72,2.50)$ & 0.45 \\
\hline Fibrinogen $(\mu \mathrm{mol} / \mathrm{l})$ & $1.44(0.85,2.45)$ & $1.67(0.90,3.11)$ & 0.10 \\
\hline E-selectin (ng/ml) & $1.49(0.89,2.51)$ & $0.96(0.52,1.80)$ & 0.84 \\
\hline ICAM-1 (ng/ml) & $1.86(1.09,3.18)$ & $1.55(0.84,2.85)$ & 0.11 \\
\hline Leptin (ng/ml) & $1.15(0.68,1.94)$ & $1.81(0.89,3.68)$ & 0.16 \\
\hline Adiponectin $(\mu \mathrm{g} / \mathrm{ml})$ & $0.90(0.53,1.52)$ & $1.22(0.66,2.25)$ & 0.59 \\
\hline sTNFR-2 (pg/ml) & $2.42(1.39,4.21)$ & $5.81(2.90,11.65)$ & $<0.001$ \\
\hline
\end{tabular}

Full multivariable models adjusted for age (continuous, years), hypertension (yes/no), BMI (continuous), ever smoked, physical activity (METS/ week), duration of type 2 diabetes (continuous, years), ACE-I/angiotensin-type 2 receptor blocker medication use (yes/no), baseline $\mathrm{HbA}_{1 \mathrm{c}}(\%)$ and eGFR in 1989. Cumulative averaged animal protein intake, alcohol intake and baseline cardiovascular disease were also considered for inclusion in the multivariable model but did not change estimates between biomarker and eGFR $\geq 25 \%$ decline and were therefore removed

ICAM-1, intracellular adhesion molecule

MET, metabolic-equivalent score

Obesity is an independent predictor of chronic kidney disease development [14]; we therefore stratified by obesity status and found that obesity modified the associations between sTNFR-2 and eGFR decline. TNF- $\alpha$ is elevated in obese compared with non-obese women and weight loss decreases these cytokine levels [15]. Because adipocytes

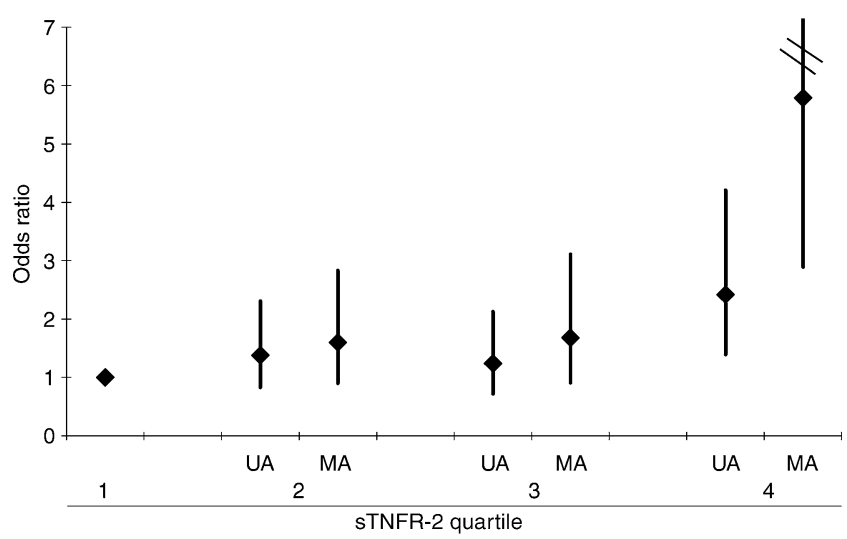

Fig. 2 Odds ratios with $95 \%$ CIs for univariate age-adjusted (UA) and multivariable-adjusted (MA) associations of quartiles of sTNFR-2 with an eGFR decline of $\geq 25 \%$ between 1989 and 2000 . Quartile 1 is the referent, $939-2,058 \mathrm{pg} / \mathrm{ml}$; quartile 2, 2,062-2,446 pg/ml; quartile $3,2,450-2,975 \mathrm{pg} / \mathrm{ml}$; quartile $4,2,979-5,758 \mathrm{pg} / \mathrm{ml}$ are now recognised as important regulators of metabolism and inflammation through their secretion of cytokines, chemokines and hormone-like proteins, we hypothesise that the larger mass of adipose tissue in obese humans may intensify the detrimental effects of elevated sTNFR-2 on kidney function.

We confirmed some of our previous findings from a cross-sectional analysis of 732 men with type 2 diabetes in the Health Professionals Follow-Up Study [4]. The strongest correlation with eGFR was seen with sTNFR-2 $(r=-0.39, p<0.001$ for men, $r=-0.28, p<0.001$ for women), whereas CRP and eGFR were weakly or not correlated $(r=-0.09, p=0.02$ for men and $r=0.03, p=0.57$ for women). No significant relations were noted between dyslipidaemia and eGFR in NHS diabetic women, whereas in the diabetic men eGFR was significantly and inversely associated with total cholesterol, triacylglycerols, non-HDL, lipoprotein(a) and apolipoprotein B. Therefore, it appears that associations between lipids and eGFR may vary by sex.

In contrast to our findings, an analysis of over 4,000 participants with type 2 diabetes in the UK Prospective Diabetes Study reported that higher baseline triacylglycerols and LDL-cholesterol were independently associated with incident renal impairment (creatinine clearance 
$<60 \mathrm{ml} / \mathrm{min}$ or doubling of plasma creatinine) [16]. The larger number of male and female participants in the UK Prospective Diabetes Study analysis allowed the examination of incident renal impairment, and the modelling approach used lipids as continuous variables rather than in quantile categories; thus, the results may not be directly comparable to the current study.

Limitations of this study include the relatively small number of women with an eGFR decline of $\geq 25 \%$ (thus limiting power for this study) and the high CVs for some of the biomarkers, which resulted in wide confidence intervals; however, the high CVs would be likely to bias the results towards the null, whereas an association with eGFR decline was observed. The participants were mostly older white women so results may not necessarily be generalisable to other populations. Although it is possible that some women with type 2 diabetes who were lost to followup would not have been included in these analyses, this would probably bias the results towards the null. Despite these limitations, this study provides important new additional information on biomarkers and kidney function in diabetes, which has not been studied extensively in humans.

We conclude that a higher level of sTNFR-2 is associated with subsequent eGFR decline in women with type 2 diabetes; the relation of sTNFR-2 with eGFR decline appeared stronger in those with a BMI of $\geq 30$. Progressive kidney disease in type 2 diabetes currently represents a major public health problem worldwide and further investigation into its pathogenesis, especially inflammatory pathways, may open new avenues for potential therapeutics. Therefore, future research may include studying whether TNF- $\alpha$ blockade is beneficial in slowing eGFR decline in type 2 diabetes.

Acknowledgements We wish to thank E. Coughlan-Gifford (Harvard School of Public Health, Boston, MA, USA) for statistical programming support and M. To (Channing Laboratory, Boston, MA, USA) for assistance in manuscript preparation. This work was supported by NIH grants K08 DK066246 (J. Lin), R03 DK078551 (J. Lin), R01DK066574 (G. C. Curhan), R01 HL065582 (F. B. Hu), R01 DK058845 (F. B. Hu), R01 DK058785 (C. Mantzoros), R01 DK079929 (C. Mantzoros) and P01 CA055075.

Duality of interest The authors declare that there is no duality of interest associated with this manuscript.

\section{References}

1. Tonelli M, Sacks F, Pfeffer M, Jhangri GS, Curhan G (2005) Biomarkers of inflammation and progression of chronic kidney disease. Kidney Int 68:237-245

2. Fried L, Solomon C, Shlipak M et al (2004) Inflammatory and prothrombotic markers and the progression of renal disease in elderly individuals. J Am Soc Nephrol 15:3184-3191

3. Sarnak MJ, Poindexter A, Wang SR et al (2002) Serum C-reactive protein and leptin as predictors of kidney disease progression in the Modification of Diet in Renal Disease Study. Kidney Int 62:2208-2215

4. Lin J, Hu FB, Rimm EB, Rifai N, Curhan GC (2006) The association of serum lipids and inflammatory biomarkers with renal function in men with type II diabetes mellitus. Kidney Int 69:336-342

5. Schulze MB, Hoffmann K, Manson JE et al (2005) Dietary pattern, inflammation, and incidence of type 2 diabetes in women. Am J Clin Nutr 82:675-684; quiz 714-715

6. National Diabetes Data Group (1979) Classification and diagnosis of diabetes mellitus and other categories of glucose intolerance. National Diabetes Data Group. Diabetes 28:1039-1057

7. Shai I, Schulze MB, Manson JE et al (2005) A prospective study of soluble tumor necrosis factor-alpha receptor II (sTNF-RII) and risk of coronary heart disease among women with type 2 diabetes. Diabetes Care 28:1376-1382

8. Pradhan AD, Rifai N, Buring JE, Ridker PM (2007) Hemoglobin A1c predicts diabetes but not cardiovascular disease in nondiabetic women. Am J Med 120:720-727

9. Stevens LA, Coresh J, Greene T, Levey AS (2006) Assessing kidney function-measured and estimated glomerular filtration rate. N Engl J Med 354:2473-2483

10. Lin J, Curhan GC (2008) Kidney function decline and physical function in women. Nephrol Dial Transplant 23:2827-2833

11. Yang T, Vesey DA, Johnson DW, Wei MQ, Gobe GC (2007) Apoptosis of tubulointerstitial chronic inflammatory cells in progressive renal fibrosis after cancer therapies. Transl Res 150:40-50

12. Khan SB, Cook HT, Bhangal G, Smith J, Tam FW, Pusey CD (2005) Antibody blockade of TNF-alpha reduces inflammation and scarring in experimental crescentic glomerulonephritis. Kidney Int 67:1812-1820

13. Nath KA (1992) Tubulointerstitial changes as a major determinant in the progression of renal damage. Am J Kidney Dis 20:1-17

14. Foster MC, Hwang SJ, Larson MG et al (2008) Overweight, obesity, and the development of stage 3 CKD: the Framingham Heart Study. Am J Kidney Dis 52:39-48

15. Ziccardi P, Nappo F, Giugliano G et al (2002) Reduction of inflammatory cytokine concentrations and improvement of endothelial functions in obese women after weight loss over one year. Circulation 105:804-809

16. Retnakaran R, Cull CA, Thorne KI, Adler AI, Holman RR (2006) Risk factors for renal dysfunction in type 2 diabetes: U.K. Prospective Diabetes Study 74. Diabetes 55:1832-1839 